\title{
SAPKs and transcription factors do the nucleocytoplasmic tango
}

\author{
Marc G. Wilkinson and Jonathan B.A. Millar ${ }^{1}$ \\ Division of Yeast Genetics, N ational Institute for Medical Research, The Ridgeway, London NW7 1AA, UK
}

A common mechanism by which eukaryotic cells sense and respond to extracel lular stimuli is via activation of a mitogen-activated protein (MAP) kinase (MAPK) cascade, which consists of three sequentially acting kinases. The M APK is activated by phosphorylation on conserved tyrosine and threonine residues by a dual-specificity MAPK kinase (M APKK or MEK). In turn, the MAPKK is activated by phosphorylation on conserved serine and threonine residues by the MAP kinase kinase kinase (MAPKKK or MEKK). The most widely studied of the MAPKs in mammalian cells is the extracellular signalregulated protein kinase (ERK) family of kinases, which are activated by a wide range of peptide growth factors and hormones (for review, see Robinson and Cobb 1997). Signal transmission from plasma membrane-associated receptors requires not only catalytic activation of these kinases but a transient spatial redistribution within the cell. Indeed, activation of both ERK 1 and ERK 2 is accompanied by their rapid relocal ization from the cytoplasm to the nucleus, and phosphorylation of target transcription factors (Chen et al. 1992; Gonzalez et al. 1993; Lenormand et al. 1993). Evidence that persistent localization of active ERK to the nucl eus increases the frequency of cellular transformation in fibroblasts, or promotes differentiation rather than proliferation in PC 12 cells, suggests that the duration of nuclear localization of active ERK is critical for the ultimate cellular response (Traverse et al. 1992, 1994; $N$ guyen et al. 1993; Dikic et al. 1994; Fukuda et al. 1996, 1997a). The mechanisms that promote nuclear accumulation of MAPKs, control the duration of nuclear local ization, or finally determine export of MAPKs back to the cytoplasm, however, are largely unknown. Furthermore, it is known that MAPKs are activated by a wide range of environmental factors, but this activation leads to very different cellular responses. This raises the question of how activated MAPK phosphorylates the correct substrate(s). In particular, how is transcriptional specificity attained by the activated MAPK such that the correct pattern of gene expression occurs in response to a specific stimulus? Two papers in this issue (Gaits et al. 1998; Toone et al. 1998) suggest that regulated and interdependent nucleo- cytoplasmic transport of both the MAPK and transcription factor play an important role in the answer to these questions.

Although most studies of MAPK activation and translocation have focused on ERKs, a new family of MAPKs recently has been identified that is activated by multiple environmental stresses, inflammatory cytokines, and certain vasoactive neuropeptides (Dérijard et al. 1994; Freshney et al. 1994; Gal cheva-Gargova et al. 1994; Han et al. 1994; Kyriakis et al. 1994; Lee et al. 1994; Rouse et al. 1994). At least two subfamilies of stress-activated MAP kinases (SAPKS) exist; the c-Jun-N (amino)-terminal kinases (JNKs) and the p38/CSBP enzymes. Pharmacological, biochemical, and genetic evidence indicates that SAPKs control a wide variety of physiological and pathol ogical conditions including development, control of cell proliferation, cell death, inflammation and response to ischemic injury. Consequently, the SAPK pathways have been attracting a great deal of attention as potential targets for novel therapeutics. Although far fewer studies of SA PK local ization have been performed, JNK does undergo nuclear translocation following UV irradiation of human fibroblasts, suggesting that similar mechanisms of nuclear translocation may be operative (Cavigelli et al. 1995). The functional dissection of SA PKs has been ai ded considerably by the identification of similar pathways in genetically tractable organisms, namely fruit flies and yeast. In particular, work from several groups has revealed a SAPK pathway in the unicellular fission yeast, Schizosaccharomyces pombe, the central elements of which are the Sty1/Spc1 MAPK and the Wis1 MAPKK (Millar et al. 1995; Shiozaki and Russell 1995; Kato et al. 1996). Importantly, the fission yeast Sty1/Spc1 M APK is activated by a similar range of environmental insults as are the mammalian SAPKs, including osmotic stress, oxidative stress, UV light, certain DN A-damaging agents, heat shock, and the protein synthesis inhibitor anisomycin (Millar et al. 1995; Shiozaki and Russell 1995; Degols et al. 1996, Degols and Russell 1997; Shieh et al. 1997).

\section{How does the MAPK go nuclear?}

In contrast to the ERKs, MEK is permenantly located in the cytopl asm (Lenormand et al. 1993; Zheng and Guan 
1994). This localization depends on a short leucinerich sequence, located in the amino terminus, called the nuclear export signal (NES), that when deleted leads to nuclear accumulation of MEK (Fukuda et al. 1996, 1997a). The NES has recently been shown to interact with the nuclear export receptor Crm1/Exportin1 in the presence of the small Ran GTPase (Fukuda et al. 1997b; Ossareh-N azari et al. 1997; Fornerod et al. 1997; Stade et al. 1997). Because Ran GAP is localized in the cytoplasm and the Ran guanine nucleotide exchange factor (GEF) RCC1 is located in the nucleus, a model has been proposed whereby RanGTP in the nucleus stimulates the formation of $\mathrm{Crm} 1 / \mathrm{NES}$ complexes leading to nuclear export. Upon translocation into the cytoplasm, Crml/ NES complexes are dissociated in the presence of RanGDP (for review, see Ullman et al. 1997). This has led to the suggestion that ERK is retained in the cytoplasm by association with its activator, MEK, and that dissociation from MEK leads to its nuclear accumulation. This model raises a number of questions that have yet to be answered satisfactorily. First, does phosphorylation of ERK cause its dissociation from MEK? Second, is ERK phosphorylation or activity necessary for nuclear import? Third, as ERK does not have a nuclear localization sequence, how does it actually enter the nucleus?

In this issue Russell and colleagues demonstrate that the fission yeast Sty1/Spc1 MAPK translocates to the nucleus in response to osmotic stress, whereas its activator, Wis1, remains cytoplasmic (Gaits et al. 1998). As is the case with other MAPKs, Sty1/Spcl is activated by dual phosphorylation on conserved threonine and tyrosine residues in subdomain VIII of the catalytic kinase domain (Millar et al. 1995; Shiozaki and Russell 1995). Russell and colleagues find that mutation of either residue inactivates the kinase and prevents its transl ocation to the nucleus. Surprisingly, when phosphorylation site mutant Sty1/Spc1 proteins are expressed in heterozygous dipl oids, which contain one fully functional copy of Sty1/Spcl, the mutant proteins remain cytoplasmic. These results indicate that SAPK activity alone is not sufficient to drive nonphosphorylatable forms of Styl/ Spcl into the nucleus. However, these experiments do not distinguish whether SAPK phosphorylation rather than SAPK activity per se is the critical determinant of nuclear rel ocal ization. To address this question, it would be necessary to know whether a phosphorylatable but catalytically inactive Sty $1 /$ Spcl is induced to undergo nuclear translocation. Regardless, these results suggest that the phosphorylated kinase must either be released from a cytoplasmic retainer and hence enter the nucleus by default, or "piggy-back" on a component of the nucleocytoplasmic transport apparatus. As Sty1/Spc1 does not accumulate in the nuclei of cells deleted for wisl, Russell and colleagues suggest that in contrast to the MEK/ERK model, Wisl is unlikely to act as a cytoplasmic anchor. However, as Sty1/Spc1 is not phosphorylated in these cells, it would not be recognized by the nucleocytoplasmic transport machinery and therefore would not accumulate in the nucleus. If release of Sty1/ Spcl from Wisl occurs independently of Styl/Spcl phosphorylation, Wisl may contribute to the retention of Sty1/Spc1 in the cytoplasm. Time will tell which of these hypotheses is correct. This still leaves the problem of how phosphorylated Sty1/Spcl actually gets into the nucleus. One is left to postulate the existence of protein(s) that promote nuclear import that can bind only the doubly phosphorylated form of the MAPK (Fig. 1). The results by Russell and colleagues contrast directly with evidence from mammalian cells that nonphosphorylated forms of ERK are still competent for nuclear entry (Gonzal ez et al. 1993; Lenormand et al. 1993). It is important to point out that the former results were obtained with mutant kinases under the control of the natural Sty1/Spc1 promoter and are therefore more likely to reflect physiological conditions than are overexpression studies using transient transfection. Alternatively, there may be an intrinsic difference between the mechanism of SAPK and ERK translocation-the former requiring dual MAPK phosphorylation that is irrelevant to the latter, or a difference between MAPK translocation in yeast and mammalian cells.

\section{MAPK nuclear retention}

Once in the nucleus, the activated MAPK must phosphorylate target proteins and then be exported back to the cytoplasm. A number of nuclear targets for the SAPKs have been described (for review, see Treisman 1996). These include the b-ZIP-containing transcription factors c-Jun and ATF2, which are components of AP-1, a dimeric transcriptional activator implicated in the control of proliferation and apoptosis (for review, see Karin 1997). Phosphorylation of both c-Jun and ATF2 by JNK kinases occurs in amino-terminally located transactivation domains and results in increased transcriptional activity (Pulverer et al . 1991; Smeal et al . 1991; Kallunki et al. 1994; Gupta et al. 1995, 1996, Livingstone et al . 1995). This occurs by direct binding of JNK kinases to specific docking domains of c-Jun and ATF2 distinct from the phosphoacceptor sites (Hibi et al. 1993; Dérijard et al. 1994; Gupta et al . 1995; Livingstone et al. 1995; Kallunki et al. 1996). A remarkable similarity between the fission yeast and mammalian SAPK pathways is highlighted by the fact that one of the direct phosphorylation targets of fission yeast Sty1/Spc1 is Atf1, a structural homolog of human ATF2 (Shiozaki and Russell 1996; Wilkinson et al. 1996). As is the case in mammalian cells, the Styl/ Spc1 SAPK binds directly to the amino terminus of Atf1 to phosphorylate it and, by inference, activate its transcriptional activity.

Surprisingly, Russell and colleagues now demonstrate that Sty1/Spcl does not accumulate in the nucleus in cells lacking Atf1. Because Atf1 is constitutively localized in the nucleus, these investigators suggest that Atf1 must act as a nuclear anchor for Sty1/Spc1 (Gaits et al. 1998). An alternative explanation is that Atf1 is required for the expression of proteins needed for MAPK import or nuclear retention. However the observation that inhibiting protein synthesis with cycloheximide prior to challenge with an osmotic stress does not block nuclear 


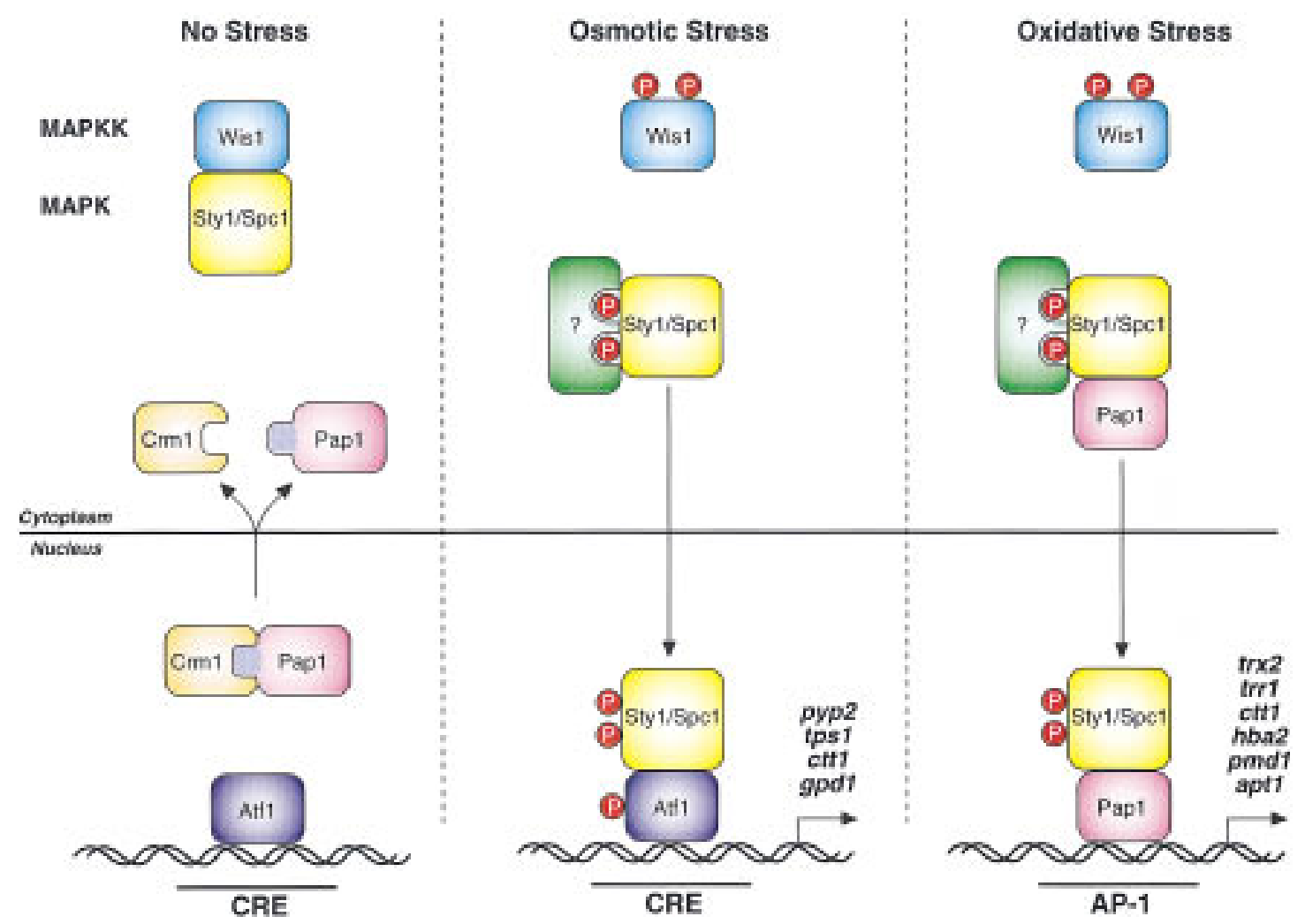

Figure 1. A speculative model for stimulus-specific gene expression by the Sty1/Spcl MAP kinase in fission yeast. The stressactivated Sty1/Spc1 M A P kinase targets two transcription factors: Atf1, a homolog of mammalian ATF2; and Pap1, a homolog of c-Jun. (Left) In unstressed cells Sty1/Spc1 and Papl are cytoplamsic, whereas Atf1 is nuclear. Pap1 is maintained in the cytoplasm via active nuclear export by Crm1/Exportin 1. (Middle) After osmotic stress Sty1/Spcl becomes doubly phosphorylated on threonine and tyrosine and enters the nucleus, possibly as a result of binding a nuclear import protein (green). Once in the nucleus Styl/Spcl phosphorylates and activates the Atf1 transcription factor to induce transcription of a subset of Atf1-dependent genes. (Right) In response to oxidative stress, Papl physically associates with doubly phosphorylated Sty1/Spcl in the cytoplasm to induce its nuclear entry but is not exported to the cytoplasm because its interaction with $\mathrm{Crm} 1 /$ Exportin is disrupted. In this case, Sty1/Spcl would be redirected to Papl-dependent promoters and cause the induction of a distinct set of genes including thioredoxin (trx2), thioredoxin reductase (trrl), and two ABC transporters (hba2 and pmd1).

accumulation of Sty1/Spc1 (P. Russell, pers. comm.), suggests that this interpretation may not be correct. To formally distinguish between these scenarios, it should be possible, based on studies of JN K and ATF2, to express an Atf1 mutant protein that is able to bind Sty1/Spc1 correctly but that is transcriptionally inactive. These studies raise the question of whether the duration of nuclear local ization depends on mechanisms control ling the interaction of MAPK and transcription factor, or on the phosphorylation state of the MAPK. Indeed, it is not known whether inactivation of the MAPK by MAPK phosphatases is a prerequisite for, or occurs as a consequence of, nuclear exit.

\section{MAPK target selection}

The studies by Russell and colleagues deal exclusively with the control of Sty1/Spcl function in response to osmotic stress (Gaits et al. 1998). However, induction of several genes, including ctt1 (catalase), trx2 (thioredoxin), and trrl (thioredoxin reductase), by oxidative stress in fission yeast requires Sty1/Spc1 but not Atf1, suggesting that Sty1/Spc1 targets an additional transcription factor. A clue to the identity of this factor was provided by the observation that induction of the same enzymes by oxidative stress in budding yeast, namely CTT1, TRX2, and TRR1, requires the AP-1 liketranscription factor Yapl. This focused attention on a c-Jun homolog in fission yeast, the Pap1 transcription factor (Toda et al. 1991). Jones and colleagues now show that Papl is specifically required for the induction of cttl, trx2, and trr1 in response to oxidative stress, and that Papl is also controlled by the Sty1/Spc1 MAPK (Toone et al. 1998). These studies further emphasize the structural and functional similarity between the fission yeast and mammalian SAPK pathways.

One is now led to ask how activated Sty $1 / \mathrm{Spcl}$ can be di rected to either Atf1-dependent or Papl-dependent promoters depending on the stimulus. A potential answer to this conundrum was provided by the observation that Yapl enters the nucl eus in response to oxi dativestress in budding yeast (Kuge and Jones 1994; Kuge et al. 1997). In 
this issue, Jones and colleagues demonstrate that Pap1 enters the nucleus when fission yeast cells are challenged with an oxidative stress. Remarkably, entry of Papl into the nucleus is abolished in cells lacking the Sty1/Spc1 M APK (T oone et al. 1998). At first sight, these observations suggest that Sty1/Spcl either phosphorylates Pap1 or a component of the nucleocytoplasmic transport machinery to induce Papl translocation to the nucleus. However, neither osmotic stress nor overexpression of the Wisl MAPKK (which fully activates Sty1/Spc1) is sufficient to drive Papl into the nucleus, indicating that activation of Sty1/Spcl alone is insufficient for Pap1 nuclear localization. This suggests that oxidative stress triggers Papl to undergo nuclear translocation by a second Styl-independent signal. In principle, relocation of a protein from the cytoplasm to the nucleus could result either from increased nuclear import or by inhibition of nuclear export. Importantly, the cysteine-rich domain (CRD) in the carboxyl terminus of budding yeast $Y$ apl contains a functional N ES that binds a homol og of the nucl ear export factor $\mathrm{Crml}$ (Kuge et al. 1997; S. Kuge, T. Toda, N. Jones, N. lizuka, and A. N omoto, in prep.). M utati ons of the conserved cysteine residues within the NES or removal of the entire sequence prevents interaction of $\mathrm{Yapl}$ with $\mathrm{Crml}$ and results in constitutive nuclear localization (S. Kuge, T. Toda, N. Jones, N . lizuka, and A. N omoto, in prep.). In one model, oxidative stress may cause a conformational change in the NES by oxidation of Cys-Cys disulfide bridges such that interaction with $\mathrm{Crm} 1$ is disrupted and nuclear export of Yapl is inhibited. Papl contains a homologous cysteine-rich N ES in its carboxyl terminus and inactivation of the fission yeast $\mathrm{Crm} 1$ homol og resul ts in nucl ear accumulation of Pap1, suggesting that Pap1 is regulated in a similar manner by the nuclear export machinery (Toda et al. 1992). Because Sty1/Spcl physically interacts with Pap1 (W. Toone, M. Samuels, and N. Jones, pers. comm.), it is possible that Sty1/Spcl carries Pap1 into the nucleus. What then would be the role of Sty1/ Spc1? As Pap1 nuclear entry is enhanced in oxidatively stressed cells overexpressing the Wis1 MAPKK, it seems likely that either dual phosphorylation or activity of Styl/Spcl contributes to the transport process. To distinguish between these possibilities it is necessary to determine whether a catalytically inactive Sty1/Spc1 that has its phosphoacceptor sites intact can support Papl entry into the nucleus.

\section{A model of stimulus-specific gene transcription}

In their simplest interpretation, the results from both the Jones group and the Russell group provide an attractive means by which interdependent nucleocytoplasmic transport of MAPKs and transcription factors determines specificity at the level of gene transcription. An attractive model is that in response to osmotic stress, doubly phosphorylated Sty1/Spc1 enters the nucleus and is preferentially directed to Atfl-dependent promoters (Fig. 1). On the other hand, oxidative stress induces a conformational change of Papl such that it can no longer bind
Crm1 but can bind to doubly phosphorylated Sty1/Spc1 in the cytoplasm. After entering the nucleus the Pap1Sty1/Spc1 complex would be redirected to Pap1-dependent promoters (Fig. 1). One can make certain predictions based on this model. If Atf1 plays no role in oxidative stress-induced gene transcription it presumably is not required either for the translocation of Papl or for the retention of Sty1/Spcl in the nucleus in response to oxidative stress. Conversely, as Papl is not required for induction of genes in response to osmotic stress, Papl is unlikely to be required for Sty $1 /$ Spcl translocation or nuclear retention in response to this stress. Unfortunately this model is likely to be too simplistic because induction of some genes by oxi dative stress, for instance, the Pyp2 MAPK phosphatase, also requires Atf1 (Wilkinson et al. 1996). Clearly many of the mechanistic details underlying these processes have yet to be unraveled, but answers may not be too far away.

It should be noted that al though Papl shares significant similarity to c-Jun, the mechanism by which Styl/ Spcl stimulates oxidative stress-induced gene transcription differs from the mechanism by which JN K activates c-Jun, in that Papl does not appear to be a direct Sty1/ Spc1 substrate and AP-1 complexes generally appear to be constitutively bound to the promoters of target genes (Rozek and Pfeifer 1993; Herr et al. 1994; W. Toone, M. Samuels, and N. Jones, pers. comm.). One is led to ask whether Sty1/Spcl plays any direct role in stimulating Papl-dependent gene transcription in addition to its role in regulating Papl nuclear entry. This is not an unreasonable notion as c-Jun can recruit JNK to phosphorylate dimerization partners via specific docking interactions (Kallunki et al.1996). At least three ATF family members have been identified in fission yeast in addition to Atf1, namely Pcr1, A tf21, and Cbp1 (Takeda et al. 1995; Kanoh et al. 1996; Shiozaki and Russell 1996; Watanabe and Yamamoto 1996; J.B.A. Millar and N. Jones, unpubl.), although it is not yet known whether any of these can serve as Sty1/Spcl substrates in vivo. Nevertheless, these proteins can potentially form a wide range of homodimers and heterodimers between themselves and Papl, each of which may have a distinct function.

The Sty1/Spc1 MAP kinase pathway, in addition to its role in controlling the cellular response to environmental stress, is required for sexual conjugation and differentiation in fission yeast. The sexual differentiation process is triggered by poor nutrient conditions that cause activation of Sty1/Spc1 (Shi ozaki and Russell 1996). Under these conditions, activation of Styl/Spcl is required for induction of the HMG box-containing transcription factor Stel1, which is critical for both sexual conjugation and meiotic progression (Shiozaki and Russell 1996; Wilkinson et al. 1996). Remarkably, induction of Stel1 also requires Atf1 and cells that lack Atf1 are sterile (Takeda et al. 1995; Kanoh et al. 1996). These results indicate that Sty1/Spcl controls the cellular differentiation process by controlling Atf1. However, Stell and the sexual diffentiation process are not induced by environmental stress even though Sty $1 / \mathrm{Spcl}$ is activated under both conditions and each is dependent on Atf1. Like- 
wise, targets of Atf1 that are induced by environmental stress, such as the Pyp2 MAPK phosphatase, are not induced by nutrient limitation (M. Wilkinson and J.B.A. Millar, unpubl.). A possible explanation for these results may also lie in the stress-specific nucleocytoplasmic transport of MAPK and transcription factor. In response to nutrient limitation, the key player may be the ATF family member Pcr1, whose functions appear limited to the sexual conjugation and differentiation process (Watananbe and Yamamoto 1996). By analogy with Papl, one could envisage a scenario in which Pcrl receives a signal initiated by nutrient limitation (possibly by lowered CAMP levels) that causes Pcr1 to bind to Sty1/Spc1 and redirect it to Pcrl/Atf1-dependent promoters. However, the observation that Pcrl is required for nuclear Iocalization of Atf1 suggests that alternative explanations are also possible (Gaits et al. 1998).

\section{Sty1/Spc1 as a model of mammalian SAPKs}

These studies emphasize that the fission yeast Styl/ Spcl kinase pathway is more closel y related to the mammalian SAPK pathway rather than the budding yeast HOG 1 pathway. Indeed, as both the mammalian and fission yeast SAPKs are activated by a similar range of environmental stimuli, one may speculate that the pathways are controlled by an evolutionarily conserved stress sensor. Curiously, despite the fact that budding yeast HOG 1 is $83 \%$ identical in amino acid sequence to Sty $1 /$ Spc1, it is activated solely by osmotic stress, the reason for which is unclear (Schüller et al. 1994). Moreover, HOG 1 targets one of two zinc finger-containing transcription factors, Msn2 and Msn4, that are unrelated to either ATF-2 or C-Jun (Schüller et al. 1994; M artinez Pastor et al. 1996). Indeed, there appear to be no ATF family members in the budding yeast genome.

Indirect evidence suggests that the similarity between fission yeast and mammalian SAPK pathways extends beyond a structural conservation of transcription factors. For example, Jones and colleagues show that two genes under the control of the Sty/Spc1-Papl pathway encode the Hba2/Bfrl and Pmd1 ATP-binding cassette (ABC) transporters. Hba2/Bfrl and Pmd1 are homologous to the human MDR1 gene (multidrug resistance gene), a $\mathrm{P}$ glycoprotein involved in energy-dependent export of drugs used in cancer chemotherapy (Higgins et al . 1992; $\mathrm{N}$ agao et al. 1995). Significantly, MDR1 is induced in mammalian cells by many of the same stresses that activate JNK and p38. It is conceivable then that inappropriate activation of SAPK pathways contributes to the multidrug resistant phenotype of many cancer cells. Inhibiting SAPK-mediated induction of ABC transporters may be an effective means of sensitizing tumorigenic cells to chemotherapeutic agents by impairing the ability of cells to exclude such drugs. A word of caution though-some cancer therapeutic agents, such as cisplatinum, that cause DNA damage can promote programmed cell death via activation of the SAPK pathways (Zanke et al. 1996). By inhibiting SAPK function one may also abrogate the ability of tumorigenic cells to un- dergo apoptosis. These observations ill ustrate the importance of understanding how the stimulus, mechanism, and duration of SAPK activation determines the ultimate cellular response. Intriguingly, fission yeast cells lacking Sty1/Spc1 are al so sensitive to DNA-damaging agents such as UV light or cis-platinum, which raises the exciting possibility that the Sty $1 /$ Spcl and SAPK pathways control a common aspect of the DNA repair process (Degols and Russell 1997; Toone et al. 1998). Further analysis of the fission yeast Sty1/Spc1 MAPK pathway will undoubtedly help us understand not only the mechani sms that determine stress-regulated nucleocytoplasmic transport but al so the regulation and function of SAPKs in various pathological and physiological conditions in mammals.

\section{Acknowledgments}

We thank Vicky Buck, Nic Jones, Humberto Martin, Paul Russell, and Takashi Toda for critical reading of the manuscript and $\mathrm{Nic}$ Jones, Shuske Kuge, and Paul Russell for communicating results prior to publication.

\section{References}

Cavigelli, M., F. Dolfi, F.X. Claret, and M. Karin. 1995. Induction of c-fos expression through JNK-mediated TCF/Elk-1 phosphorylation. EMBO J. 14: 5957-5964.

Chen, R.H., C. Sarnecki, and J. Blenis. 1992. N uclear localization and regulation of erk- and rsk-encoded protein kinases. Mol. Cell. Biol. 12: 915-927.

Degols, G. and P. Russell. 1997. Discrete roles of the Spcl kinase and the Atf1 transcription factor in the UV response of Schizosaccharomyces pombe. Mol. Cell. Biol. 17: 33563363.

Degols, G., K. Shiozaki, and P. Russell. 1996. Activation and regulation of the Spcl stress-activated protein kinase in Schizosaccharomyces pombe. Mol. Cell. Biol. 16: 28702877.

Dérijard, B., M. Hibi, I.-H. Wu, T. Barrett, B. Su, T. Deng, M. Karin, and R.J. Davis. 1994. JNK1: A protein kinase stimulated by UV light and $\mathrm{Ha}$-Ras that binds and phosphorylates the c-jun activation domain. Cell 76: 1025-1037.

Dikic, I., J. Schlessinger, and I. Lax. 1994. PC12 cells overexpressing the insulin receptor undergo insulin-dependent neuronal differentiation. Curr. Biol. 4: 702-708.

Fornerod, M., M. Ohno, M. Yoshida, and I.W. Mattaj. 1997. CRM 1 is an export receptor for leucine-rich nuclear export signals. Cell 90: 1051-1060.

Freshney, N.W., L. Rawlinson, F. Guesdon, E. Jones, S. Cowley, J. Hsuan, and J. Saklatvala. 1994. Interleukin-1 activates a novel protein kinase cascade that results in the phosphorylation of hsp27. Cell 78: 1039-1049.

Fukuda, M ., I. Gotoh, Y. Gotoh, and E. N ishida. 1996. Cytoplasmic localization of mitogen-activated protein kinase kinase directed by its $\mathrm{N} \mathrm{H}$ 2-terminal, leucine-rich short amino acid sequence, which acts as a nuclear export signal. J. Biol. Chem. 271: 20024-20028.

Fukuda, M., I. Gotoh, M. Adachi, Y. Gotoh, and E. Nishida. 1997a. A novel regulatory mechanism in the mitogen-activated protein (MAP) kinase cascade. J. Biol. Chem. 272: 32642-32648.

Fukuda, M., S. A sano, T. N akamura, M. Adachi, M. Yoshida, M. 
Yanagida, and E. Nishida. 1997b. CRM 1 is responsible for intracellular transport mediated by the nuclear export signal. Nature 390: 308-311.

Gaits, F., G. Degols, K. Shiozaki, and P. Russell. 1998. Phosphorylation and association with the transcription factor Atf1 regulate localization of $\mathrm{Spcl}$ stress-activated kinase in fission yeast. Genes \& Dev. (this issue).

Galcheva-Gargova, Z., B. Dérijard, I.-H. Wu, and R.J. Davis. 1994. An osmosensing signal transduction pathway in mammalian cells. Science 265: 806-808.

Gonzalez, F.A., A. Seth, D.L. Raden, D.S. Bowman, F.S. Fay, and R.J. Davis. 1993. Serum-induced translocation of mitogenactivated protein kinase to the cell surface ruffling membrane and the nucleus. J. Cell Biol. 122: 1089-1101.

Gupta, S., D. Campbell, B. Dérijard, and R.J. Davis. 1995. Transcription factor ATF2 regulation by the JNK signal transduction pathway. Science 267: 389-393.

Gupta, S., T. Barrett, A.J. Whitmarsh, J. Cavanagh, H.K. Sluss, B. Dérijard, and R.J. Davis. 1996. Selective interaction of JNK protein kinase isoforms with transcription factors. EMBO J. 15: $2760-2770$.

Han, J., J.-D. Lee, L. Bibbs, and R.J. Ulevitch. 1994. A MAP kinase targeted by endotoxin and hyperosmolarity in mammalian cells. Science 265: 808-811.

Herr, I., H. van Dam, and P. Angel. 1994. Binding of promoterassociated AP-1 is not altered during induction and subse quent repression of the c-jun promoter by TPA and UV irradiation. Carcinogenesis 15: 1105-1113.

Hibi, M., L. Anning, T. Smeal, A. Minden, and M. Karin. 1993. Identification of an oncoprotein- and UV-responsive protein kinase that binds and potentiates the c-Jun activation domain. Genes \& Dev. 7: 2135-2148.

Higgins, C.F. 1992. ABC transporters: From microorganisms to man. Annu. Rev. Cell Biol. 8: 67-113.

Kallunki, T., T. Deng, M. Hibi, and M. Karin. 1996. c-Jun can recruit JNK to phosphorylate dimerization partners via specific docking interactions. Cell 87: 929-939.

Kallunki, T., B. Su, I. Tsigelny, H.K. Sluss, B. Dérijard, G. M oore, R. Davis, and M. Karin. 1994. JN K2 contains a specificity-determining region responsible for efficient c-Jun binding and phosphorylation. Genes \& Dev. 8: 2996-3007.

Kanoh, J., Y. Watanabe, M. Ohsugi, Y. Iino, and M. Yamamoto. 1996. Schizosaccharomyces pombe gad $7^{+}$encodes a phosphoprotein with a bZIP domain, which is required for proper $\mathrm{G}_{1}$ arrest and gene expression under nitrogen starvation. Genes Cells 1: 391-408.

Karin, M., Z.-G. Liu, and E. Zandi. 1997. AP-1 function and regulation. Curr. Opin. Cell Biol. 9: 240-246.

Kato, T., K. Okazaki, H. M urakami, S. Stettler, P. Fantes, and H. Okayama. 1996. Stress signal, mediated by a HOG1-like MAP kinase, controls sexual development in fission yeast. FEBS Lett. 378: 207-212.

Kuge, S. and N. Jones. 1994. YAP1 dependent activation of TRX2 is essential for the reponse of Saccharomyces cerevisiae to oxidative stress by superoxides. EMBO J. 13: 655664.

Kuge, S., N. Jones, and A. N omoto. 1997. Regulation of yAP-1 nucl ear localization in response to oxidative stress. EMBO J. 16: $1710-1720$.

Kyriakis, J.M., P. Banerjee, E. Nikolakaki, T. Dai, E.A. Rubie, M.F. A hmad, J. A vruch, and J.R. Woodgett. 1994. The stressactivated protein kinase family of c-jun kinases. Nature 369: 156-160.

Lee, J.C., J.T. Laydon, P.C. M cDonnell, T.F. Gallagher, S. Kumar, D. Green, D. McNulty, M.J. Blumenthal., J.R. Heys, S.W. Landvatter, J.E. Strickler, M.M. McLaughlin, I.V. Sie- mens, S.M. Fisher, G.P. Livi, J.R. White, J.L. Adams, and P.R. Young. 1994. A protein kinase involved in the regulation of inflammatory cytokine biosynthesis. N ature 372: 739-746.

Lenormand, P., C. Sardet, G. Pages, G. L'Allemain, A. Brunet, and J. Pouyssegur. 1993. Growth factors induce nuclear translocation of MAP kinases (p42mapk and p44mapk) but not of their activator MAP kinase kinase (p45mapkk) in fibroblasts. J. Cell Biol. 122: 1079-1088.

Livingstone, C., G. Patel, and N. Jones. 1995. ATF-2 contains a phosphorylation-dependent transcriptional activation domain. EMBO J. 14: 1785-1797.

Martinez Pastor, M.T., G. Marchler, C. Schüller, A. Marchler Bauer, H. Ruis, and F. Estruch. 1996. The Saccharomyces cerevisiae zinc finger proteins Msn2p and Msn4p are re quired for transcriptional induction through the stress response element (STRE). EMBO J. 15: 2227-2235.

Millar, J.B.A., V. Buck, and M.G. Wilkinson. 1995. Pyp1 and Pyp2 PT Pases dephosphorylate an osmosensing M AP kinase controlling cell size at division in fission yeast. Genes \& Dev. 9: 2117-2130.

Nagao, K., Y. Taguchi, M. Arioka, H. Kadokura, A. Takatsuki, K. Yoda, and M. Yamasaki. 1995. bfr1t, a novel gene of Schizosaccharomyces pombe which confers brefeldin A resistance, is structurally related to the ATP-binding cassette superfamily. J. Bacteriol. 177: 1536-1543.

N guyen, T.T., J.C. Scimeca, C. Filloux, P. Peraldi, J.L. Carpentier, and E. Van Obberghen. 1993. Co-regulation of the mitogen-activated protein kinase, extracellular signal-regulated kinase 1, and the 90-kDa ribosomal S6 kinase in PC12 cells. Distinct effects of the neurotrophic factor, nerve growth factor, and the mitogenic factor, epidermal growth factor. J. Biol. Chem. 268: 9803-9810.

Ossareh-N azari, B., F. Bachelerie, and C. Dargemont. 1997. Evidence for a role of CRM 1 in signal-mediated nuclear protein export. Science 278: 141-144.

Pulverer, B.J., J.M. Kyriakis, J. Avruch, E. Nikolakaki, and J.R. Woodgett. 1991. Phosphorylation of c-jun mediated by M AP kinases. Nature 353: 670-674.

Robinson, M.J. and M.H. Cobb. 1997. Mitogen-activated protein kinase pathways. Curr. Opin. Cell Biol. 9: 180-186.

Rouse, J., P. Cohen, S. Trigon, M. M orange, A. Alonso-Llamazares, D. Zamanillo, T. Hunt, and A. N ebreda. 1994. A novel kinase cascade triggered by stress and heat shock that stimulates MAPKAP kinase-2 and phosphorylation of the small heat shock proteins. Cell 78: 1027-1037.

Rozek, D. and G.P. Pfeifer. 1993. In vivo protein-DNA interactions at the c-jun promoter: preformed complexes mediate the UV response. Mol. Cell. Biol. 13: 5490-5499.

Schüller, C., J.L. Brewster, M.R. Alexander, M.C. Gustin, and H. Ruis. 1994. The HOG pathway controls osmotic regulation of transcription via the stress response el ement (STRE) of the Saccharomyces cerevisiae CTT1 gene. EMBO J. 13: 43824389.

Shieh, J.-C., M.G. Wilkinson, V. Buck, B. Morgan, K. Makino, and J.B.A. Millar. 1997. The M cs4 response regulator coordinately controls the stress activated Wak1-Wis1-Sty1 M AP kinase pathway and fission yeast cell cycle. Genes \& Dev. 11: 1008-1022.

Shiozaki, K. and P. Russell. 1995. Cell cycle control linked to extracellular environment by MAP kinase pathway in fission yeast. Nature 378: 739-743.

-_- 1996. Conjugation, meiosis, and the osmotic stress response are regulated by Spc1 kinase through Atf1 transcription factor in fission yeast. Genes \& Dev. 10: 2276-2288.

Smeal, T., B. Binetruy, D.A. M ercola, M. Birrer, and M. Karin. 1991. Oncogenic and transcriptional cooperation with Ha- 
Ras requires phosphorylation of c-Jun on serines 63 and 73. Nature 354: 494-496.

Stade, K., C.S. Ford, C. Guthrie, and K. Weis. 1997. Exportin 1 (Crmlp) is an essential nuclear export factor. Cell 90: 10411050.

Takeda, T., T. Toda, K. Kominami, A. Kohnosu, M. Yanagida, and $\mathrm{N}$. Jones. 1995. Schizosaccharomyces pombe atf1 ${ }^{+}$encodes a transcription factor required for sexual development and entry into stationary phase. EMBO J. 14: 6193-6208.

Toda, T., M. Shimanuki, and M. Yanagida. 1991. Fission yeast genes that confer resistance to staurosporine encode an AP1-like transcription factor and a protein kinase related to the mammalian ERK1/MAP2 and budding yeast FUS3 and KSS1 kinases. Genes \& Dev. 5: 60-73.

Toda, T., M. Shimanuki, Y. Saka, H. Yamano, Y. Adachi, M. Shirakawa, Y. Kyogoku, and M. Yanagida. 1992. Fission yeast pap1-dependent transcription is negatively regulated by an essential nuclear protein, crm1. Mol. Cell. Biol. 12: 5474-5484.

Toone, W.M., S. Kuge, M. Samuels, B.A. Morgan, T. Toda, and $N$. Jones. 1998. Regulation of the fission yeast transcription factor Pap1 by oxidative stress: Requirement for the nuclear export factor Crml (Exportin) and the stress activated M AP kinase Styl. Genes \& Dev. (this issue).

Traverse, S., N. Gomez, H. Paterson, C. M arshall, and P. Cohen. 1992. Sustained activation of the mitogen-activated protein (MAP) kinase cascade may be required for differentiation of PC 12 cells. Comparison of the effects of nerve growth factor and epidermal growth factor. Biochem. J. 288: 351-355.

Traverse, S., K. Seedorf, H. Paterson, C.J. Marshall, P. Cohen, and A. Ullrich. 1994. EGF triggers neuronal differentiation of PC 12 cells that overexpress the EGF receptor. Curr. Biol. 4: 694-701.

Treisman, R. 1996. Regulation of transcription by MAP kinase cascades. Curr. O pin. Cell Biol. 8: 205-215.

Ullman, K.S., M.A. Powers, and D.J. Forbes. 1997. N uclear export receptors: from importin to exportin. Cell 90: 967-970.

Watanabe, Y. and M. Yamamoto. 1996. Schizosaccharomyces pombe pcr $^{+}$encodes a CREB/ATF protein involved in regulation of gene expression for sexual differentiation. Mol. Cell. Biol. 16: 704-714.

Wilkinson, M.G., M. Samuels, T. Takeda, T. Toda, M.W. Toone, J.-C. Shieh, J.B.A. Millar, and N.C. Jones. 1996. The Atf1 transcription factor is a target for the Sty 1 stress activated MAP kinase pathway in fission yeast. Genes \& Dev. 10: 2289-2301.

Zanke, B.W., K. Boudreau, E. Rubie, E. Winnett, L.A. Tibbles, L. Zon, J. Kyriakis, F.-F. Liu, and J.R. Woodgett. 1996. The stress activated protein kinase pathway mediates cell death following injury induced by cis-platinum, UV irradiation of heat. Curr. Biol. 6: 606-613.

Zheng, C.F. and K.-L. Guan. 1994. Cytoplasmic localization of the mitogen-activated protein kinase activator MEK. J. Biol. Chem. 269: 19947-19952. 


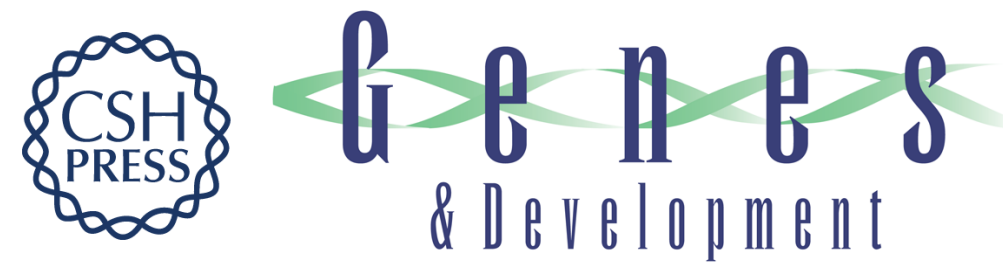

\section{SAPKs and transcription factors do the nucleocytoplasmic tango}

Marc G. Wilkinson and Jonathan B.A. Millar

Genes Dev. 1998, 12:

Access the most recent version at doi:10.1101/gad.12.10.1391

References This article cites 55 articles, 25 of which can be accessed free at: http://genesdev.cshlp.org/content/12/10/1391.full.html\#ref-list-1

License

Email Alerting Receive free email alerts when new articles cite this article - sign up in the box at the top Service right corner of the article or click here.

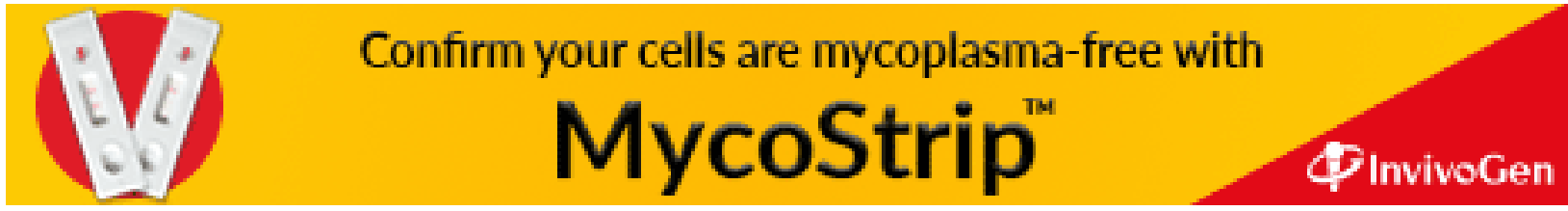

